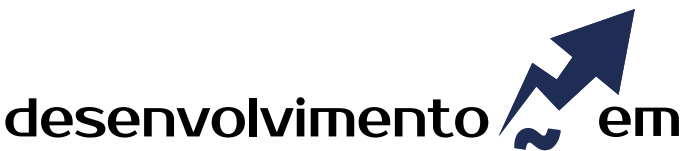 QUESTÃO
}

\section{Fatores Facilitadores e Restritivos à Cooperação Universidade e Empresa: O Caso Udesc}

http://dx.doi.org/10.21527/2237-6453.2018.45.273-291

Recebido em: 17/3/2017

Aceito em: 18/4/2018

\author{
Marcelo Gomes Cardoso ${ }^{1}$, Nério Amboni², \\ Gerson Volney Lagemann ${ }^{3}$, Rui Otávio Bernardes de Andrade ${ }^{4}$
}

\begin{abstract}
RESUMO
O objetivo deste estudo é analisar os principais fatores facilitadores e restritivos no desenvolvimento de projetos de pesquisa da Universidade do Estado de Santa Catarina (Udesc) em cooperação com empresas, segundo o olhar de 10 líderes de grupos de pesquisa e de 14 gestores de empresas. A base teórica do estudo compreende a discussão dos argumentos de autores nacionais e internacionais acerca da cooperação Universidade-Empresa (U-E). A pesquisa é descritiva com abordagem qualitativa. As técnicas de coleta de dados utilizadas foram a análise documental e o questionário, com os dados sendo tratados de modo descritivo. Os fatores "recursos financeiros adicionais" e "acesso a pesquisadores qualificados" foram considerados os fatores mais facilitadores segundo os líderes de grupos de pesquisa e gestores de empresas, respectivamente; e o "excesso de burocracia" como o fator mais restritivo à cooperação Universidade-Empresa, tanto para os líderes de grupos de pesquisa quanto para os gestores de empresas. Os achados são corroborados por estudos teóricos e empíricos nacionais e internacionais, reforçando os avanços da literatura e das questões metodológicas utilizadas. Conclui-se que a universidade deverá aperfeiçoar as práticas de cooperação com as empresas, estabelecendo políticas institucionais que possibilitem a redução da burocracia e dos prazos de tramitação dos processos, a fim de minimizar as barreiras identificadas na interação e possibilitar o desenvolvimento de mais pesquisas aplicadas entre os envolvidos na cooperação. O estudo tem como limitações a volatilidade das percepções dos pesquisados, a área geográfica de Santa Catarina e a delimitação em três Centros de Ensino da Udesc. Palavras-chave: Cooperação. Universidade. Empresa. Inovação. Udesc.
\end{abstract}

\section{FACILITATING AND RESTRICTIVE FACTORS ON COOPERATION BETWEEN UNIVERSITY AND COMPANY:} THE UDESC CASE

\begin{abstract}
The purpose of this study is to analyze the main facilitators and restrictive factors in the development of research projects at the Santa Catarina State University (Udesc) in cooperation with companies, according to the perspective of 10 research groups leaders and 14 companies' managers. The theoretical basis of the study includes the discussion of the arguments of national and international authors about the university-companies cooperation (U-E). The research is descriptive with qualitative approach. The data collection techniques used were document analysis and questionnaire, being the data treated descriptively. Factors "additional financial resources" and "access to qualified researchers" were considered as the most facilitating factors according to research groups leaders and companies's managers, respectively; and the "excessive bureaucracy" as the most limitating factor to university-business cooperation, not only to leaders of research groups but also to the companies' managers. The findings were supported by national and international theoretical and empirical studies, reinforcing the progress of literature and the mentioned methodological questions. It follows that the university should improve cooperation practices with companies, establishing institutional policies that enable the reduction of bureaucracy and deadlines of procedures in order to minimize the barriers identified in the interaction and encourage the development of more applied research among those involved in the cooperation. The study has certain limitations such as the volatility of the perceptions of respondents, the geographical area of Santa Catarina State and the delimitation in three learning centers of Udesc.
\end{abstract}

Keywords: Cooperation. University. Company. Innovation. Udesc.

\footnotetext{
${ }^{1}$ Mestrado Profissional em Administração pela Universidade do Estado de Santa Catarina. Administrador e técnico universitário de desenvolvimento na Universidade do Estado de Santa Catarina.marcelocardososc@gmail.com

${ }^{2}$ Doutorado em Engenharia de Produção pela Universidade Federal de Santa Catarina. Professor da Universidade do Estado de Santa Catarina. ambony30@yahoo.com.br

${ }^{3}$ Doutorado em Administração pela Universidade Federal da Bahia. Professor da Universidade do Estado de Santa Catarina. gerson.lagemann@udesc.br

${ }^{4}$ Doutorado em Engenharia de Produção pela Universidade Federal de Santa Catarina. Professor da Universidade do Grande Rio. andrade@ novanet.com.br
} 
No amplo horizonte do Ensino Superior, a universidade se projeta como um ambiente de saber e como um centro aglutinador e multidisciplinar na produção de conhecimento de ciência e tecnologia. Embora não haja uma visão unívoca sobre a concepção de universidade e suas funções, já em 1935 Teixeira (1962) alertava que a universidade não tem uma função única e exclusiva.

O processo evolutivo da universidade foi marcado por duas revoluções inter-relacionadas. A "primeira revolução acadêmica" consistiu na inclusão da pesquisa na universidade, ao lado da docência/ensino, consolidando o papel desta como uma parte indissociável do processo de ensino. A "segunda revolução acadêmica" foi desencadeada pelo envolvimento da universidade com o setor produtivo, além do ensino e da pesquisa (WEBSTER; ETZKOWITZ, 1991). Este estreitamento das relações foi fortalecido com a crise do fordismo nos anos 70 (CLARKE, 1991) e com a adoção do modelo alemão (ótica científico-profissional) por algumas universidades norte-americanas, como Princeton, Michigan, Harvard e Stanford (MAIA, 2005). A partir dos anos 80 a cooperação é intensificada, despertando curiosidade e atenção de investigadores, decisores políticos e profissionais, principalmente por melhorar as capacidades de pesquisa, difundir a inovação e aumentar empregabilidade dos estudantes das Instituições de Ensino Superior (ETZKOWITZ, 2004; RANGA et al. 2013).

Segundo Durham (1990), a Cooperação Escola-Empresa trata-se de um desenvolvimento inevitável, que resulta da transformação do sistema produtivo e da natureza do trabalho de investigação científica. A crescente relevância da pesquisa para o sistema produtivo e a necessidade do uso da tecnologia na própria pesquisa tornam essa conexão necessária.

Autores como Mathis (2001), Lopes (2000), Goebel e Miura (2004) concluíram que as universidades são promotoras fundamentais para a consolidação do processo de desenvolvimento local e regional. Schneider (2002) analisa a universidade como um atrativo de grande importância para o surgimento de novas atividades e investimentos nos municípios nos quais elas são instaladas, devido ao volume considerável de recursos injetados, seja por meio dos salários dos docentes e dos técnico-administrativos, seja mediante o consumo efetuado pelos estudantes, o que desencadeia um efeito multiplicador para a economia do município. Goebel e Miura (2004) entendem que a universidade incentiva o desenvolvimento, gerando emprego e renda, como também prepara profissionais para atuarem como multiplicadores de conhecimentos em qualquer setor da sociedade. A cooperação entre universidades e empresas, segundo Noveli e Segatto (2012), tem desempenhado um papel significativo nas inovações tecnológicas, contribuindo para o desenvolvimento econômico e como incentivo à produção científica sobre o tema.

Para Feng et al. (2015), os estudos sobre a cooperação Universidade-Empresa (universidade-indústria) vêm sendo feitos desde o ano de 1966, tendo no ano de 2008 o maior número de publicações sobre o assunto (41), correspondendo a $7 \%$ do total das 587 publicações feitas no período entre 1966-2013. Segundo esses autores, 25 revistas publicaram 4 ou mais artigos sobre colaboração U-E, entre as quais sobressaem: "American Chemical Society" (41 publicações), evidenciando uma forte relação da indústria química com a academia; "Research Policy" (33); "Technovation" (17); "Science" (16); "Higher Education" (12); "IEEE Transactions on Education" (12); "Scientometrics" (12) 
e "Nature" (11). Dessas publicações, 20\% estão concentradas em 25 autores, entre os quais destacam-se Leydesdorff (14 publicações) e Etzkowitz (9 publicações). O período entre $2005-2012$ concentrou cerca de $40 \%$ dessas pesquisas.

Da mesma forma, no âmbito do Brasil, a pesquisa desenvolvida por Rosa et al. (2015) ratifica a intensificação e o interesse de acadêmicos e profissionais acerca do tema cooperação U-E. A Figura 1 revela que 62,75\% das publicações brasileiras sobre cooperação U-E, nos últimos 14 anos, estão concentradas nos últimos seis anos (20092014). Treze revistas publicaram 3 ou mais artigos sobre colaboração U-E, e, entre estas, destacam-se: Revista de Administração da USP (34 publicações), o periódico com o maior número de publicações (25\%), seguida da Revista Brasileira de Inovação (18 publicações) e da Revista de Administração da Inovação (13 publicações). Esses três periódicos representam quase $50 \%$ das publicações sobre cooperação U-E no Brasil entre os anos de 1984 e 2014.

Figura 1 - Publicações brasileiras sobre cooperação U-E nos periódicos científicos de Administração entre os anos 1984 e 2014

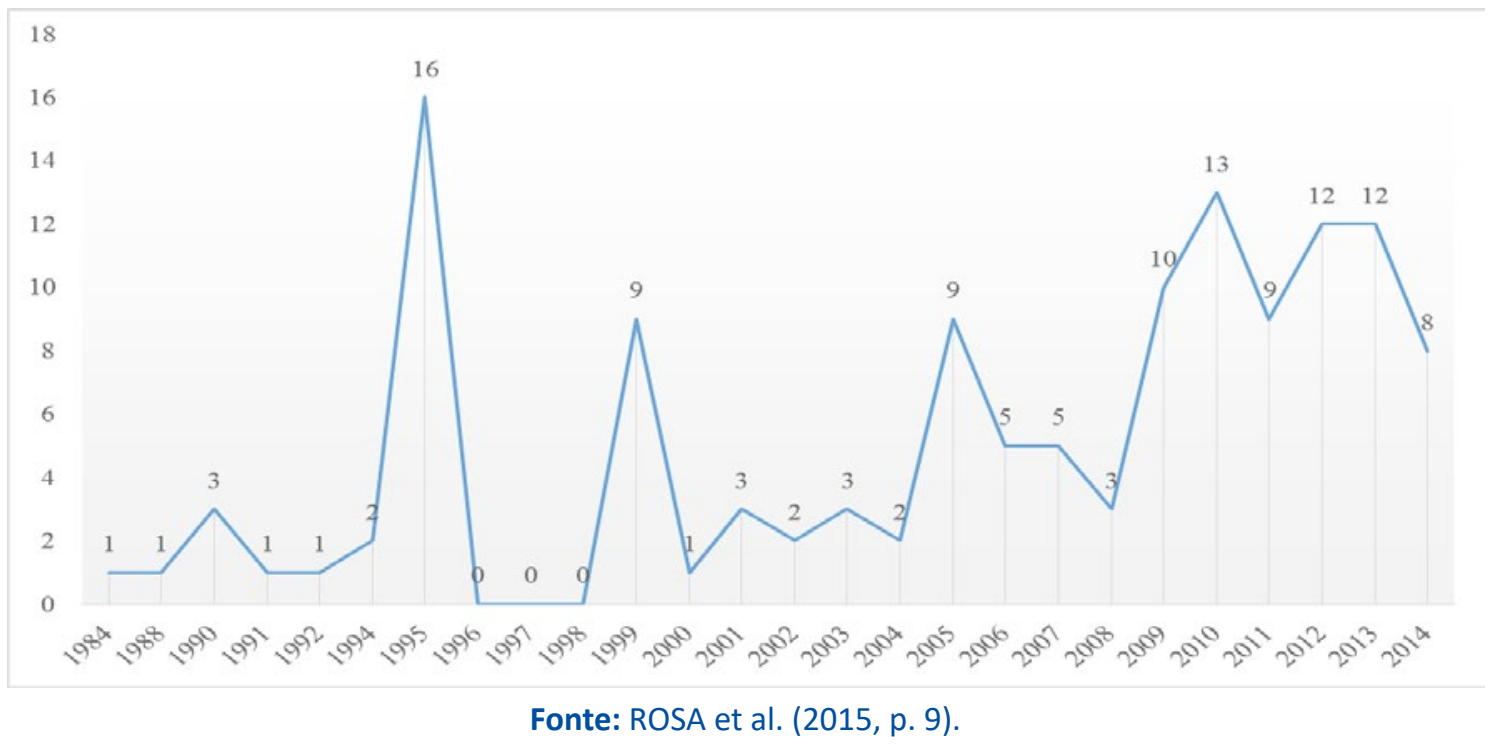

Os argumentos apresentados e a produção acadêmica sobre o tema demonstram a relevância da cooperação Universidade-Empresa, quer para as universidades (por exemplo: fonte de receitas públicas e privadas; renda de licenciamento de patentes; renda adicional ou benefício financeiro para pesquisadores; estímulo ao avanço tecnológico e/ou atividades de investigação em certas áreas estratégicas; aquisição ou acesso a equipamentos; criação de oportunidades de negócios e contribuição de desenvolvimento econômico para a região) quer para as empresas (por exemplo: novos produtos e/ou processos; produtos e/ou processos de melhoria; patentes, protótipos, propriedade intelectual; melhoria da competitividade; acesso a subvenções públicas; aumento da velocidade de inovação para o mercado e criação de riqueza) (ANKRAH; AL-TABBAA, 2015).

A cooperação Universidade-Empresa proporciona diferentes benefícios, porém em muitas situações estes não são concretizados, devido às barreiras impostas, tanto pelas universidades como pelas empresas. Para Ribeiro e Andrade (2008, p. 73) as universidades e as empresas são entidades diferentes e diferem significativamente na na- 
tureza e objeto de suas atividades, pois as universidades buscam a qualidade das pesquisas e a ampla divulgação dos resultados obtidos, enquanto as empresas visam ao lucro e, para tal, selecionam criteriosamente os projetos nos quais deverão se engajar segundo sua potencialidade comercial, risco e retorno econômico-financeiro.

Nesta direção, este artigo tem por objetivo analisar os principais fatores facilitadores e restritivos no desenvolvimento de projetos de pesquisa em três Centros de Ensino da Udesc que cooperam com empresas no ano de 2015: Centro de Ciências Agroveterinárias - CAV - Lages (pecuária); Centro de Ciências Tecnológicas - CCT - Joinville (indústria) e o Centro de Ciências da Administração e Socioeconômicas - Esag - Florianópolis (comércio e serviços). No desenvolvimento do artigo, além dos argumentos, fundamentos e resultados de pesquisas apresentados, são destacados os fatores que facilitam e restringem esta relação. Em seguida são descritos os procedimentos metodológicos. $\mathrm{Na}$ sequência os resultados são apresentados, discutidos e confrontados com os achados de pesquisas já realizadas por autores nacionais e internacionais. Por fim, são apresentadas as conclusões e as referências.

\section{REFERENCIAL TEÓRICO}

Cooperação trata-se de uma ação social articulada e alinhavada por objetivos comuns, visando à solução de problemas concretos (CAMARGO, 1960). A cooperação é o eixo estruturante para a sustentabilidade da sociedade contemporânea, como contraponto à competição, e se constitui num dos componentes básicos da estratégia das organizações no seu processo de desenvolvimento institucional (PLONSKI, 1998; KOROTKA, 2015).

A cooperação U-E é um modelo de arranjo interinstitucional de organizações de natureza fundamentalmente distinta, que podem ter diferentes tipos (por exemplo: atividades ligadas a P\&D, serviços e capacitação), modalidades (por exemplo: convênios entre U-E) e mecanismos (por exemplo: pesquisas tecnológicas em parceria) (PLONSKI, 1998; PORTO, 2004).

As fases envolvidas na cooperação U-E foram alvo de pesquisas de vários pesquisadores, entre os quais cabe mencionar as discutidas por Plewa et al. (2013): 1) pré-linkage: identificação das pessoas ou equipes com potenciais parceiros de pesquisa; 2) establishment: identificação de interesses e necessidades de cada uma das partes; 3 ) engagement: estabelecimento do ambiente cooperativo; 4) advancement: sustentabilidade do relacionamento e; 5) latente phase: continuidade, cooperação futura.

A cooperação U-E, por ser um processo complexo e multidimensional e por envolver múltiplas partes interessadas, está "longe" de ser um processo tranquilo, principalmente devido às diferenças estruturais e de objetivos entre os agentes, podendo gerar expectativas e percepções contraditórias, desmotivando os professores no desenvolvimento de projetos de pesquisa de cunho mais aplicado (SEGATTO, 1996; PORTO, 2004; SILVEIRA, 2005; PHILBIN, 2011). A cooperação também é verificada dentro da própria universidade como resultante de pesquisas multi e interdisciplinares, colaborando no desenvolvimento da visão sistêmica e na busca de soluções para problemas específicos. 
A cooperação U-E, dependendo das parcerias, metas, objetivos e de outros interesses, pode proporcionar diferentes benefícios: 1) reunir recursos e potencializar oportunidades, incentivando projetos de inovação para apoiar o desenvolvimento tecnológico (METCALFE, 2010; PERKMANN; NEELY; WALSH, 2011); 2) incentivar o desenvolvimento de novas pesquisas que venham a contribuir para o processo de melhoria ou inovação de produtos, processos e serviços (BENEDETTI; TORKOMIAN, 2010; DIAS; PORTO, 2013; FERREIRA; RAMOS, 2015); 3) promover a internacionalização, trazendo contribuições sociais e econômicas significativas (LE, 2015); 4) estimular a troca de informações entre os agentes envolvidos; 5) melhorar a qualidade da pesquisa e do ensino por meio da aprendizagem em situações concretas (PERKMANN; NEELY; WALSH, 2011); 6) reunir novas ideias para futuras pesquisas e adquirir novas inspirações para resolução de problemas (KAYMAZ; ERYIĞI, 2011).

Midlej e Fialho (2005, p. 172), analisando a relação existente entre universidade e região, entendem que a universidade tende a ocupar uma posição fundamental nessa dinâmica, empreendendo processos de inovação tecnológica, de produção e difusão da ciência e cultura, ocupando lugar estratégico no desenvolvimento socioeconômico, qualificando os diferentes níveis de ensino do próprio sistema educacional, além de desempenhar uma pluralidade de funções em termos de formação acadêmico-profissional.

De acordo com Quandt $(2009,2012)$, a busca por novos conhecimentos e competências fora da organização ajuda as empresas a se tornarem mais competitivas e inovadoras. E este processo multidimensional, associado à convergência de conhecimentos complementares e processos de interação, auxiliam tanto no desenvolvimento econômico das organizações que cooperam quanto no desenvolvimento econômico da região em que estas organizações estão localizadas. A cooperação deve ser estimulada para facilitar o aprendizado mútuo e reforçar as relações de confiança que são construídas ao longo do tempo no sistema produtivo local (QUANDT, 2012).

Freitas e Freitas (2013), na mesma linha, comentam que as relações sinérgicas constituídas entre organizações locais na promoção de ações conjuntas orientadas para o desenvolvimento local tornam-se importantes na medida em que vão consolidando bases sociais de cooperação, formando o tecido social no qual essas organizações se alicerçam, potencializando a mobilização de recursos, pessoas e ações para uma proposta compartilhada de transformação da realidade social e econômica. Transformar conhecimento em produtos inovadores implica a articulação entre instituições, cidades, regiões e atores sociais (Rolim; Serra, 2009).

D'Este e Patel (2007), Perkmann e Schildt (2014) e Franco e Haase (2015) afirmam que a cooperação entre as universidades e as empresas incentiva o desenvolvimento de inovações tecnológicas, de produtos, processos, gestão e de novas habilidades e competências na busca de vantagens competitivas. Estes autores consideram a cooperação U-E como ligações bidirecionais a fim de permitir a difusão de criatividade, ideias, habilidades e o intercâmbio de pessoas, visando à criação de valor mútuo ao longo do tempo.

Diante de todas as possibilidades de mecanismos de cooperação, vários fatores de ordem interna e externa atuam como facilitadores e restritivos à cooperação Universidade-Empresa. 


\section{Fatores Facilitadores e Restritivos à Cooperação Universidade-Empresa}

Pelo fato de a cooperação U-E ser um processo complexo e multidimensional, envolvendo múltiplas partes interessadas nos níveis contextuais, organizacionais, grupais e individuais, inúmeros fatores de ordem interna e externa podem tanto incentivar quanto inibir a cooperação.

O estudo realizado por Segatto (1996) em relação ao processo de cooperação tecnológica Universidade-Empresa demonstrou que as principais motivações percebidas pelos entrevistados em relação às universidades foram as seguintes: recursos financeiros adicionais; recursos materiais adicionais; realização da função social da universidade; prestígio que será obtido pelo pesquisador; divulgação da imagem da universidade; obtenção de conhecimentos práticos sobre os problemas existentes e incorporação de novas informações aos processos de ensino e pesquisa universitários. Já os fatores que mais motivam as empresas são: acesso a recursos humanos altamente qualificados da universidade; redução dos custos e/ou riscos envolvidos nos projetos de pesquisa e desenvolvimento; acesso aos mais novos conhecimentos desenvolvidos no meio acadêmico; identificação de alunos da instituição de ensino para recrutamento futuro e resolução dos problemas técnicos que geraram a necessidade da pesquisa cooperativa.

No entender de Carvalho (1997, p. 345), entre os fatores indicados como facilitadores, destacam-se: contato com pessoal técnico das empresas; necessidade de sucesso nos projetos; política do departamento/curso para a cooperação; estrutura de equipamentos e laboratórios disponíveis na instituição e política da instituição para a cooperação com empresa.

Segatto (1996), no estudo realizado em relação ao processo de cooperação tecnológica Universidade-Empresa, identificou que, entre as principais barreiras estão a burocracia universitária e a duração muito longa do projeto. Estes fatores restritivos também são apontados por Carvalho (1998, p. 345), que afirma que "entre os fatores indicados como limitadores, destacam-se: I) burocracia institucional e II) carga horária didática padrão".

Segundo Alvim (1998, p. 106), tanto por parte das universidades como por parte das empresas, existem barreiras à cooperação que precisam ser superadas, tais como: barreiras organizacionais; barreiras pessoais/profissionais e barreiras culturais. Lemos (2013) comenta que a falta de incentivos em direção ao estabelecimento de relações com empresas tem gerado um sentimento de descontentamento, tanto por parte dos alunos quanto em relação aos professores, formando uma barreira para a realização de interações.

Em estudo mais recente, Ankrah e AL-Tabbaa (2015) destacam alguns fatores que podem dificultar a colaboração entre Universidade-Empresa. Os obstáculos oriundos da universidade são, entre outros, os seguintes: ameaças à autonomia das pesquisas; acordos de confidencialidade podem bloquear a disseminação do conhecimento; abandono de pesquisas básicas de longo prazo em favor apenas das aplicadas e orientadas para os resultados de curto prazo; departamentos universitários atuando apenas como extensões para a solução de problemas das empresas, mediante contratos que exigem soluções de curto prazo. Na visão das empresas, os obstáculos são: as burocracias aca- 
dêmicas fazem com que o processo seja lento, podendo atrasar a comercialização de tecnologias e o cumprimento de objetivos da empresa; o retorno sobre o capital investido pode ser lento; aumento das despesas administrativas com a contratação de pessoal para gerenciar o processo de cooperação com a universidade.

A cooperação U-E ao mesmo tempo que é incentivada, também é limitada por vários fatores de ordem interna e externa. Como alternativa para minimizar a situação Ripper Filho, citado por Silva (2010), destaca que tanto as universidades quanto as empresas têm a ganhar com a parceria, mas que a dinâmica do processo de interação deve ser mais bem compreendida por elas para não se criar falsas expectativas e que, embora tenham objetivos bastante diferenciados, universidade, institutos e empresas são parte de um sistema e devem interagir de forma a maximizar os benefícios para os seus objetivos.

Na mesma linha, Porto (2006) ressalta que os objetivos de cada uma das partes envolvidas e do projeto como um todo devem estar estabelecidos, para se evitar resultados instáveis e caóticos. Da mesma forma, para Santana e Porto (2009) o setor empresarial deve modificar sua postura reativa, ou seja, de ficar esperando que a universidade faça todo o trabalho de criação das condições ideais para que haja a interação entre as partes.

No item subsequente são abordados os procedimentos metodológicos que serviram de orientação no desenvolvimento da pesquisa.

\section{PROCEDIMENTOS METODOLÓGICOS}

A instituição universitária, objeto do estudo, Universidade do Estado de Santa Catarina - Udesc - caracteriza-se, sobretudo, por ser pública e notabilizada nacionalmente pela qualidade, tendo sido considerada pelo Ministério da Educação (MEC), no Índice Geral de Cursos (IGC), das instituições de educação superior em 2013, como a quarta melhor universidade estadual do Brasil, a 18a no geral, entre 192 instituições avaliadas, e a melhor em Graduação, em Santa Catarina (UNIVERSIDADE..., 2014).

Em 2015, a Udesc conta com 12 Centros de Ensino, 29 Polos de EAD, 54 cursos de Graduação, 10 Especializações, 29 Mestrados, 11 Doutorados e 158 Grupos de Pesquisa (UNIVERSIDADE..., 2017). A partir de uma busca feita no Diretório dos Grupos de Pesquisa do CNPq (CONSELHO..., 2015) é possível observar que apenas 20 destes 158 Grupos de Pesquisa (GPs) cooperam com empresas; 138 GPs não possuem atividades de cooperação com empresas, equivalendo a $87 \%$ deste total. Apenas $13 \%$ do total de GP possuem cooperação.

Do total dos 20 GPs da Udesc que cooperam com empresas, os autores escolheram 12 GPs de Centros de Ensino localizados em regiões com características peculiares do Estado de Santa Catarina, a saber: Centro de Ciências Agroveterinárias - CAV - Lages (agropecuária); Centro de Ciências Tecnológicas - CCT - Joinville (indústria) e o Centro de Ciências da Administração e Socioeconômicas - Esag - Florianópolis (comércio e serviços). Desta forma, as unidades de análise compreendem os Centros de Ensino e as unidades de observação, ou seja, os líderes de pesquisa que cooperam com empresas (Quadro 1). 
Quadro 1 - Centros de Ensino e grupos de pesquisa analisados

\begin{tabular}{|l|c|c|c|c|}
\hline $\begin{array}{l}\text { Centros de Ensino da Udesc pesquisados } \\
\text { Total de grupos de pesquisa por Centro de Ensino }\end{array}$ & $\begin{array}{c}\text { CAV } \\
\text { CCT }\end{array}$ & $\begin{array}{c}\text { ESAG } \\
\text { Total }\end{array}$ & $\begin{array}{c}13 \\
65\end{array}$ \\
\hline $\begin{array}{l}\text { Total de grupos de pesquisa por Centro de Ensino que cooperam } \\
\text { com empresas }\end{array}$ & 04 & 07 & 01 & 12 \\
\hline $\begin{array}{l}\text { Total de líderes de grupos de pesquisa que cooperam com } \\
\text { empresas e que responderam ao questionário }\end{array}$ & 03 & 06 & 01 & 10 \\
\hline Total de empresas que cooperam com os grupos de pesquisa & 05 & 11 & 04 & 20 \\
\hline $\begin{array}{l}\text { Total de gestores de empresas que cooperam com os grupos de } \\
\text { pesquisa e que responderam ao questionário }\end{array}$ & 03 & 09 & 02 & 14 \\
\hline
\end{tabular}

Fonte: Elaborado pelos autores.

Nos três Centros de Ensino da Udesc pesquisados foram identificados 65 Grupos de Pesquisa no total. Destes 65, 12 cooperam com empresas. Dos 12 Grupos de Pesquisa que cooperam nos três Centros de Ensino, 10 responderam ao questionário desta pesquisa, equivalendo a $83 \%$ do total de Grupos de Pesquisa que cooperam com empresas nestes três Centros de Ensino.

As técnicas de coleta de dados utilizadas foram a análise documental e questionário, complementados pela vivência e experiência de um dos pesquisadores deste artigo como gestor no Núcleo de Inovação Tecnológica (NIT) da Udesc. O processo de coleta de dados primários (pesquisa empírica) foi realizado da seguinte forma: a) pesquisa na Internet no site do Diretório de Grupos de Pesquisa do CNPq (CONSELHO..., 2015); b) elaboração do questionário a partir dos fatores facilitadores e restritivos identificados na revisão bibliográfica e na busca sistemática; c) pré-teste com quatro grupos de pesquisa e quatro empresas; d) análise crítica das questões por assunto pesquisado; e) ajustes dos questionários; f) contato telefônico com os líderes dos grupos de pesquisa e gestores das empresas; g) encaminhamento dos questionários via on-line pelo Google forms; h) gestão e recebimento dos questionários preenchidos; i) organização e tratamento descritivo e qualitativo; j) identificação e descrição dos fatores facilitadores, restritivos e demais sugestões.

O processo descritivo tem por objetivo identificar e registrar os grupos de pesquisa e os principais fatores facilitadores e restritivos no desenvolvimento de projetos de pesquisa da Universidade do Estado de Santa Catarina (Udesc) em cooperação com empresas, segundo o olhar de 10 líderes de grupos de pesquisa e de 14 gestores de empresas (Quadro 1). O tratamento dos dados é feito de modo qualitativo.

As informações e os dados foram descritos, interpretados e triangulados à luz dos argumentos teóricos e práticos e dos achados de pesquisas já desenvolvidas sobre o tema cooperação Universidade-Empresa. Os autores selecionaram na descrição e interpretação dos dados os 8 fatores facilitadores e os 8 fatores restritivos mais citados pelos líderes de grupos de pesquisa e pelos gestores das empresas pesquisadas.

\section{Fatores Facilitadores e Restritivos na Cooperação Universidade e Empresa: o Caso Udesc}

Neste bloco são apresentados os 8 principais fatores identificados nesta pesquisa como facilitadores na cooperação U-E (Figuras 2 e 3) e os 8 principais fatores identificados como restritivos (Figuras 4 e 5), na visão dos líderes dos grupos de pesquisa e gestores de empresas. 
Figura 2 - Fatores Facilitadores na Visão dos Líderes de Grupos de Pesquisa

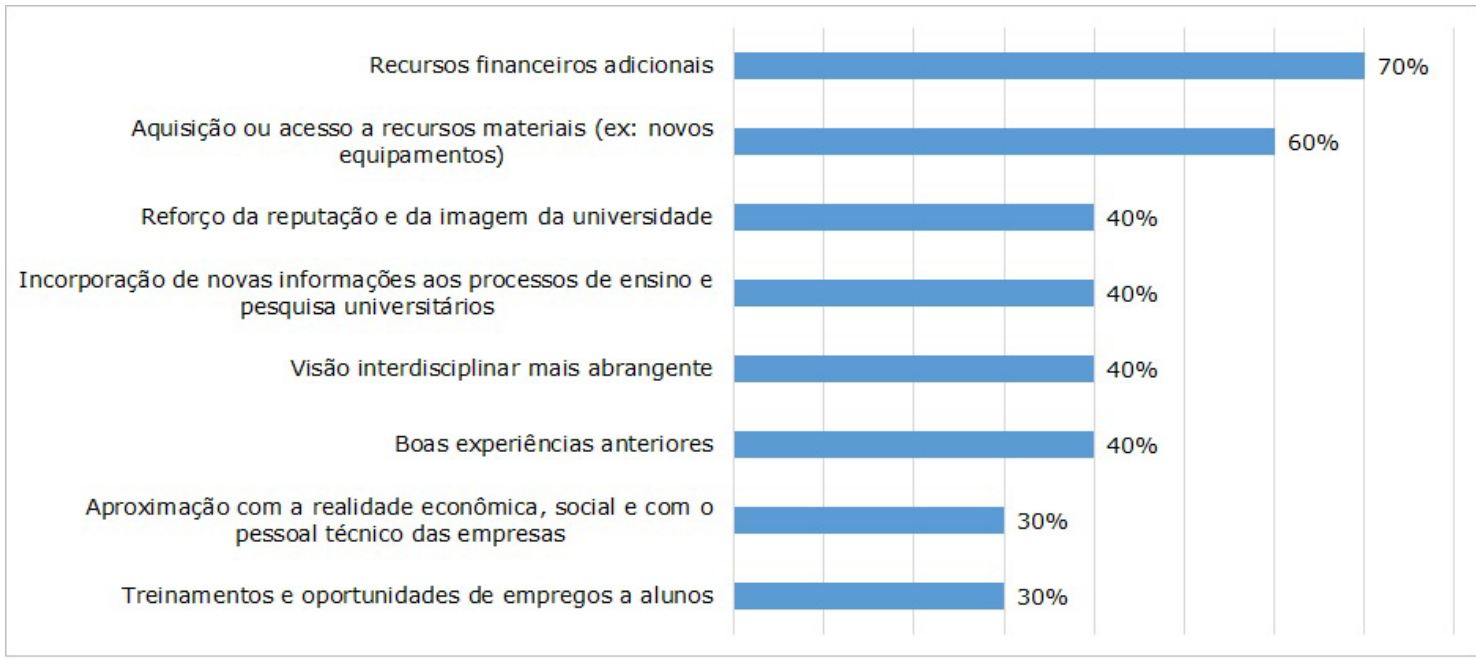

Fonte: Elaborado pelos autores.

O fator facilitador mais citado pelos líderes de grupos de pesquisa, entre os oito selecionados, foi Recursos financeiros adicionais (70\%). O relacionamento com as empresas tem sido uma alternativa importante para as universidades na busca de recursos que sirvam para financiar bolsas de estudos, adquirir equipamentos e material de consumo, montar laboratórios e formar grupos de pesquisa multidisciplinares (SEGATTO, 1996). Closs e Ferreira (2012, p. 423) destacam a "necessidade de recursos adicionais, melhor utilização de equipamentos e maior interação com o mercado". A possibilidade de as universidades obterem recursos financeiros é um fator comum para a receptividade em cooperar com empresas. As cooperações com empresas geram, segundo Ankrah e Al-Tabbaa (2015, p. 12), "fontes de receitas públicas e privadas" e "renda adicional (ou benefício financeiro) para os pesquisadores".

A Aquisição ou acesso a recursos materiais (ex: novos equipamentos) foi considerado por $60 \%$ dos pesquisados. Na visão de Segatto-Mendes e Mendes (2006, p. 66), "no termo aditivo relativo à cooperação entre PUC-PR e Electrolux, cabia à empresa o fornecimento de equipamentos (refrigeradores, freezer, pacotes de carga térmica e cargas de refrigerante R-134a entre outros) e recursos financeiros liberados mensalmente". Ankrah e Al-Tabbaa (2015, p. 12) destacam a "aquisição ou acesso a equipamentos" como benefício institucional resultante da cooperação U-E. O Reforço da reputação $e$ da imagem da universidade foi considerado por $40 \%$ dos pesquisados, confirmando os achados das pesquisas realizadas por Segatto (1996), Dan (2013) e Ankrah e Al-Tabbaa (2015), pois nas cooperações entre U-E há a divulgação da imagem e o reforço da reputação da universidade na comunidade como um todo.

A Incorporação de novas informações aos processos de ensino e pesquisa universitários e a Visão interdisciplinar mais abrangente também foram destacadas como fatores facilitadores na realização da cooperação U-E, ambos por $40 \%$. A cooperação com as empresas surge como "importante alternativa de articulação entre o conhecimento tecnológico e a demanda por novas tecnologias. É nesse ambiente interdisciplinar que o docente poderá se aperfeiçoar tanto em conteúdo como em procedimentos" (CARVALHO, 1997, p. 2). A cooperação U-E ajuda a "estimular o avanço tecnológico e/ou atividades de investigação em certas áreas estratégicas" (ANKRAH; AL-TABBAA, 2015, p. 12). 
As Boas experiências anteriores é outro fator facilitador, também considerado por $40 \%$ dos pesquisados. Stal e Fujino (2005, p. 14), no entanto, alertam que se as experiências anteriores forem negativas, pode haver a desistência da cooperação por parte das empresas, principalmente devido "à falta de comprometimento da equipe, à falta de sigilo e não cumprimento de prazos", por parte da universidade. Para Guarnica e Torkomian (2009, p. 634), "experiência boa de relacionamento prévio entre as partes envolvidas" é um dos fatores de apoio na transferência de tecnologia entre U-E. Closs e Ferreira (2012, p. 423) argumentam que "[...] boas experiências de relacionamentos anteriores [...]" é um dos fatores facilitadores na interação U-E.

Os fatores Aproximação com a realidade econômica, social e com o pessoal técnico das empresas e os Treinamentos e oportunidades de empregos a alunos foram considerados por $30 \%$, respectivamente. A participação de engenheiros de empresas em cursos de Mestrado favorece a comunicação, entrosamento e aproximação dos objetivos acadêmico e empresarial (CLOSS; FERREIRA, 2012), assim como a situação mais comum para estabelecimento de contatos com as empresas ocorre a partir de alunos ou ex-alunos da universidade conhecidos dos professores e que possuem vínculo com tais empresas (LEMOS, 2013). Também treinamentos e oportunidades de empregos a alunos representam benefícios que podem ser gerados com a colaboração U-E (ANKRAH; AL-TABBAA, 2015).

Figura 3 - Fatores Facilitadores na Visão dos Gestores de Empresas

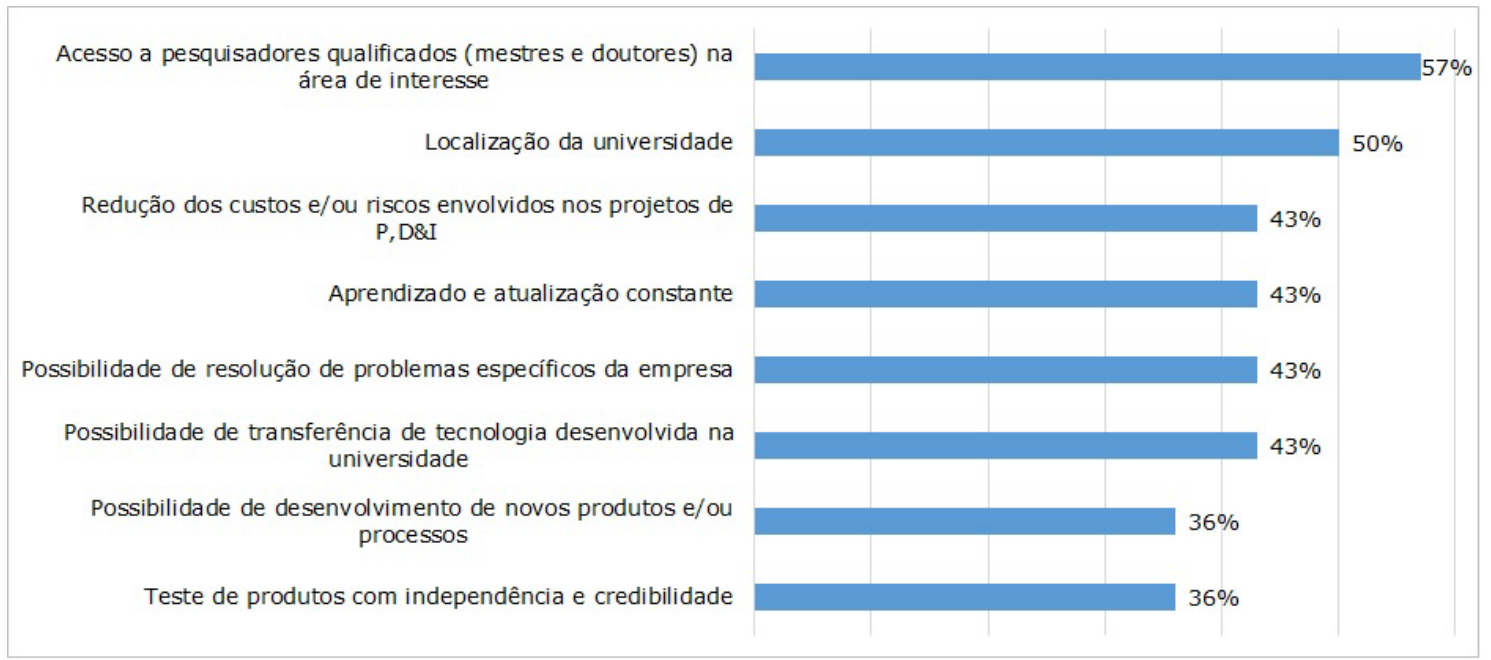

Fonte: Elaborado pelos autores.

De outro lado, na visão dos gestores de empresas pesquisados, o fator facilitador mais citado é o Acesso a pesquisadores qualificados (mestres e doutores) na área de interesse (57\%). Um dos motivadores centrais para as empresas, na cooperação tecnológica U-E, é o acesso a recursos humanos com elevada qualificação (SEGATTO, 1996; SEGATTO-MENDES; MENDES, 2006; SILVA, 2015). Ankrah e Al-Tabbaa (2015) destacam a variedade de conhecimentos e pesquisa multidisciplinar; o acesso à consultoria especializada e à oportunidade para acessar uma rede mais ampla de competências.

A Localização da universidade é o segundo fator facilitador mais citado pelos gestores de empresas (50\%). Etzkowitz (1998, p. 829) afirma que "a proximidade geográfica faz a diferença no sentido de incentivar a interação adequada". Os fatores fa- 
cilitadores seguintes identificados citados por $43 \%$ dos gestores de empresas, respectivamente, foram: a Redução dos custos e/ou riscos envolvidos nos projetos de P,D\&l; - Aprendizado e atualização constante; a Possibilidade de resolução de problemas específicos da empresa e a Possibilidade de transferência de tecnologia desenvolvida na universidade.

Em relação à Redução dos custos e/ou riscos envolvidos nos projetos de $P, D \& l$, observa-se que ao repartir custos e riscos entre as duas instituições, a pesquisa cooperativa possibilita maior investimento na geração de novas tecnologias voltadas a produtos e processos que garantam maior competitividade às organizações e a ampliação do conhecimento científico da nação (SEGATTO-MENDES; MENDES, 2006). Também, os recursos financeiros disponibilizados pelo governo para estimular as pesquisas desenvolvidas nas universidades, em parceria com empresas, vão ao encontro da necessidade de redução de custos e riscos por parte das empresas (CLOSS; FERREIRA, 2012).

O Aprendizado e a atualização constante decorrente da transformação tecnológica impõe investimentos permanentes em tecnologia, e isto torna clara a necessidade de aprendizado contínuo (BOTELHO; CARRIJO; KAMASAKI, 2007; CLOSS; FERREIRA, 2012). A possibilidade de resolução de problemas específicos da empresa é reforçada por Segatto (1996) e Ankrah e Al-Tabbaa (2015), pois a resolução de problemas técnicos é um motivador central para a empresa. Na USP, o "Disque-Tecnologia" fornece consultas a empresários, e isso tem ajudado a solucionar problemas específicos de natureza tecnológica, administrativa, gerencial, mercadológica, de aprimoramento profissional, das relações de trabalho e de difusão cultural (SILVA, 2015).

O outro fator facilitador identificado foi a Possibilidade de transferência de tecnologia desenvolvida na universidade. A transferência de tecnologia está evoluindo, principalmente porque as empresas estão se aproximando das universidades, muitas vezes estimuladas pelos financiamentos à pesquisa que têm sido concedidos pelos agentes financiadores (TORKOMIAN, 1997). As universidades dispõem de invenções tecnológicas patenteadas que podem ser transferidas para as empresas, buscando estimular a inovação e, consequentemente, a criação de empregos e a elevação da renda, quando bem-sucedida (MAIA, 2005; CLOSS; FERREIRA, 2012; LEMOS, 2013).

A Possibilidade de desenvolvimento de novos produtos e/ou processos e o Teste de produtos com independência e credibilidade foram considerados fatores facilitadores por $36 \%$ dos gestores de empresas. As empresas obtêm várias vantagens com a integração com a universidade, como maior facilidade para o desenvolvimento de novos produtos (MAIA, 2005). Na visão de Ankrah e Al-Tabbaa (2015, p.12), "novos produtos e/ou processos" são benefícios econômicos que podem ser gerados pelas empresas ao colaborarem com as universidades. Em relação ao fator Teste de produtos com independência e credibilidade, Maia (2005, p. 113) o considera como "serviços contratados (desenvolvimento de protótipos, testes, etc.)" nas relações institucionais formais realizadas por meio de convênios. Closs e Ferreira (2012) argumentam que entre os vários tipos de interação U-E está o "uso de laboratórios pelas empresas, para pesquisas e testes" e Ankrah e Al-Tabbaa (2015) destacam o "Teste de produtos com independência e credibilidade" como benefício institucional para as empresas durante o processo de colaboração com as universidades. 
Da mesma forma que a interação U-E é motivada, também é inibida por vários fatores de ordem interna e externa, conforme demonstrado nas Figuras 4 e 5.

Figura 4 - Fatores Restritivos na Visão dos Líderes de Grupos de Pesquisa

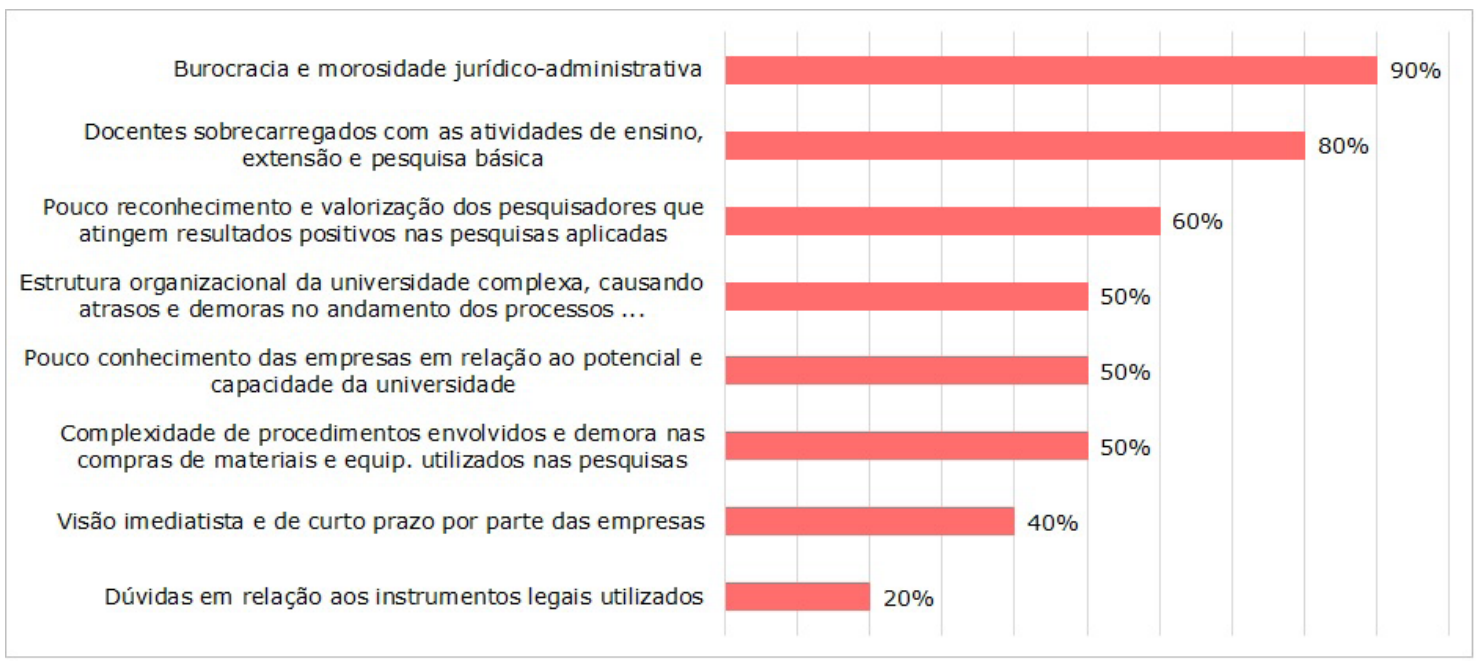

Fonte: Elaborado pelos autores.

O fator restritivo Burocracia e morosidade jurídico-administrativa foi citado por 90\% dos líderes de grupos de pesquisa, pelo fato de a burocracia estar inibindo a realização de parcerias com as empresas. O excesso de burocracia, ligado aos aspectos legais e formais, é uma das principais barreiras no processo de cooperação U-E. Ela também pode atrasar a comercialização de tecnologias e o cumprimento de objetivos da empresa, podendo gerar conflitos e conduzir o processo à baixa produtividade (SEGATTO, 1996; SANTANA; PORTO, 2009; CLOSS; FERREIRA, 2012; ANKRAH; AL-TABBAA, 2015).

O segundo fator restritivo, Docentes sobrecarregados com as atividades de ensino, extensão e pesquisa básica, foi citado por $80 \%$ dos líderes de grupos de pesquisa. 0 fator está relacionado ao plano de trabalho do professor da Udesc, regulamentado pela Resolução 029/2009 do Conselho Universitário da Udesc.

Como argumento de defesa, os docentes recorrentemente expunham a sobrecarga de atividades como um fator que pode fazer com que eles atrasem alguma ação que Ihes cabia no processo de transferência de tecnologia, mais precisamente, o patenteamento. Foi exposto por eles que suas funções na universidade (ensino, pesquisa e extensão) já preenchem completamente o seu tempo. Tal fato é ainda mais enfatizado, quando foi exposto uma quarta atividade docente, a administrativa, função amplamente desempenhada pelos professores/pesquisadores, especialmente por não contarem com equipes que os apoiem em tal tipo de atividade (SANTANA; PORTO, 2009, p. 422).

O terceiro fator restritivo Pouco reconhecimento e valorização dos pesquisadores que atingem resultados positivos nas pesquisas aplicadas foi citado por $60 \%$ e a Estrutura organizacional da universidade complexa, causando atrasos e demoras no andamento dos processos de cooperação com empresas foi mencionado por $50 \%$ dos líderes de grupos de pesquisa. A estrutura organizacional da universidade é inadequada para a prestação de serviços (ALVIM, 1998; VIEIRA; VIEIRA, 2004; SANTANA; PORTO, 2009). As três dimensões da atividade acadêmica universitária - ensino, pesquisa e extensão - 
vêm se tornado reféns de um processo burocrático incontrolável, submetido a normas e dependências muitas vezes desnecessárias produzidas pelas estruturas piramidais de apoio (CLOSS; FERREIRA, 2012).

O Pouco conhecimento das empresas em relação ao potencial e capacidade da universidade e a Complexidade de procedimentos envolvidos e a demora nas compras de materiais e equipamentos utilizados nas pesquisas foram citados por $50 \%$ líderes de grupos de pesquisa, respectivamente, como fatores restritivos na cooperação U-E. O desconhecimento do potencial e das capacidades instaladas na universidade representa uma das barreiras encontradas pelo setor produtivo durante a cooperação U-E (ALVIM, 1998). A principal queixa dos pesquisadores sobre a quantidade e complexidade de procedimentos envolvidos nas atividades de pesquisa, notadamente aqueles relacionados às questões de funcionamento do grupo de pesquisa, tais como a compra de materiais e equipamentos, são as barreiras de transação (LEMOS, 2013).

A Visão imediatista e de curto prazo por parte das empresas também foi considerada um fator restritivo para $40 \%$ dos líderes de grupos de pesquisa. 0 empresário brasileiro precisa alterar a sua postura e acreditar mais nas capacidades das universidades, atuando em pesquisas de prazos mais longos e com menor imediatismo de resultados (SEGATTO, 1996; ALVIM, 1998; MAIA, 2005). As Dúvidas em relação aos instrumentos legais utilizados foi o oitavo fator restritivo identificado, citado por $20 \%$ dos líderes de grupos de pesquisa. A falta de uma legislação clara dificulta o desenvolvimento das atividades, confirmando os achados de Tecchio et. al. (2013, p. 9):

Mesmo com a criação da Lei no 10.973 (Lei da Inovação) e da Lei no 11.196, o processo não está claro e a falta de uma legislação interna, específica para nortear o processo, se torna uma dificuldade para o estabelecimento de projetos em cooperação, o que é corroborado por Mendes e Sbragia (2002), quando expõem que a ausência de instrumentos legais que regulamentem as atividades de pesquisa envolvendo universidades e empresas, concomitantemente, acabam dificultando o processo.

A seguir são apresentados os fatores restritivos na visão dos gestores de empresas.

Figura 5 - Fatores Restritivos na Visão dos Gestores de Empresas

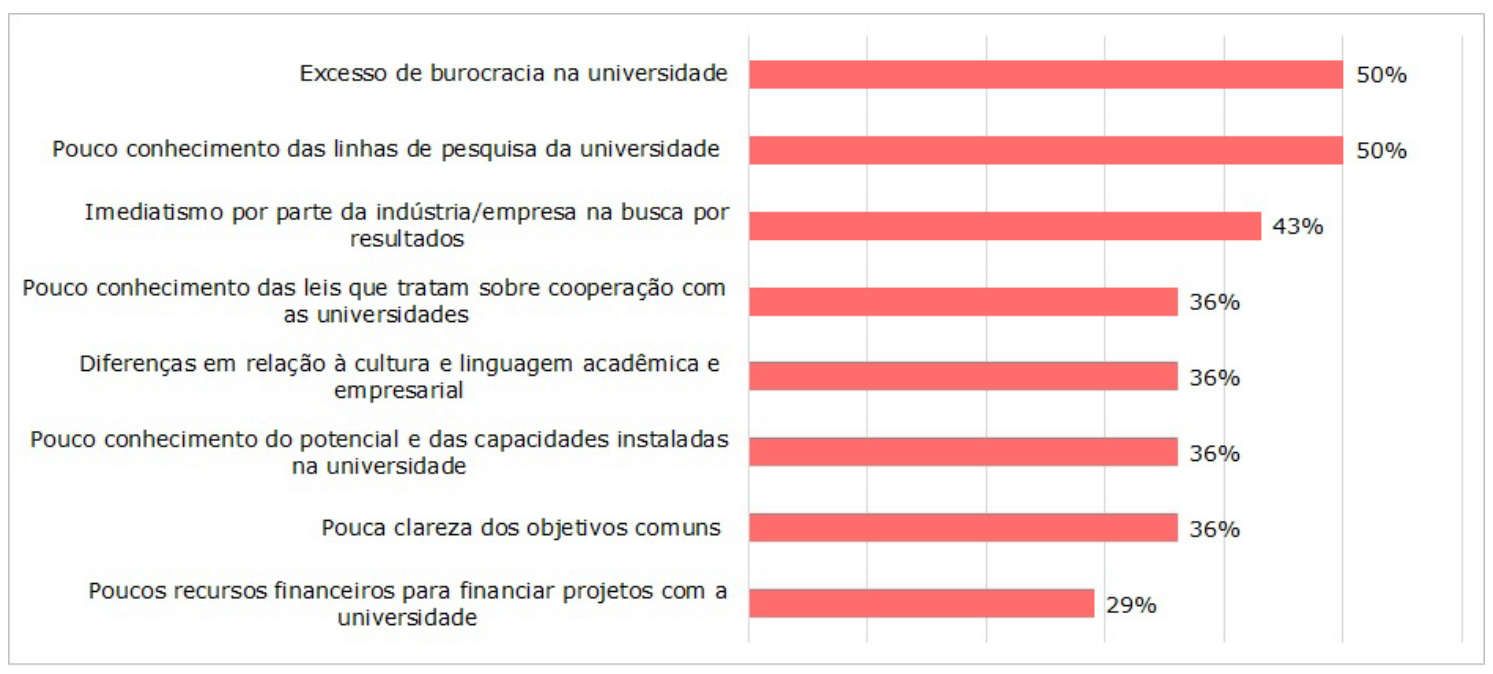

Fonte: Elaborado pelos autores. 
No momento em que são considerados os resultados obtidos com os gestores de empresas, nota-se que dos oito fatores restritivos citados, quatro deles também foram mencionados pelos líderes de grupos de pesquisa e já comentados, quais sejam: a) Excesso de burocracia na universidade, citado por $50 \%$ dos gestores de empresas e por 90\% líderes de grupos de pesquisa; b) Pouco conhecimento do potencial e das capacidades instaladas na universidade, citado por $36 \%$ dos gestores de empresas e por $50 \%$ dos líderes de grupos de pesquisa; c) Imediatismo por parte da indústria/empresa na busca por resultados, citado por $43 \%$ dos gestores de empresas e por $40 \%$ dos líderes de grupos de pesquisa; d) Pouco conhecimento das leis que tratam sobre cooperação com as universidades foi outro fator restritivo identificado, citado por $36 \%$ dos gestores de empresas e por $20 \%$ dos líderes de grupos de pesquisa.

Além do Excesso de burocracia na universidade, citado por $50 \%$ dos gestores de empresas, o Pouco conhecimento das linhas de pesquisa da universidade também foi outro fator restritivo citado por $50 \%$ dos gestores que participaram da pesquisa. Os fatores restritivos Diferença em relação à cultura e linguagem acadêmica e empresarial e a Pouca clareza dos objetivos comuns foram citados por $36 \%$ dos gestores de empresas. A questão cultural é motivada pela diferença de nível de conhecimento entre as pessoas da universidade e da empresa. As empresas brasileiras ainda não têm a cultura de utilizar as universidades e os institutos de pesquisa como fontes de tecnologia (SEGATTO-MENDES; MENDES, 2006; SANTANA; PORTO, 2009; CLOSS; FERREIRA, 2012). A imprecisão dos objetivos do trabalho contratado com a empresa está entre os aspectos negativos mais apontados na cooperação U-E (SANTANA; PORTO, 2009; BENEDETTI; TORKOMIAN, 2010). O oitavo fator restritivo na visão de $29 \%$ dos gestores de empresas é ter Poucos recursos financeiros para financiar projetos com a universidade. Em relação a este fator, destaca-se que esta pesquisa identificou que $50 \%$ das empresas questionadas investiram, nos últimos 2 anos, de $2 \%$ a $5 \%$ do faturamento em P\&D; $25 \%$ das empresas investiram de $1 \%$ a $2 \% ; 17 \%$ das empresas investiram menos de $1 \%$; e $8 \%$ das empresas investiram mais de $5 \%$ do faturamento em P\&D; nenhuma empresa disse que "não investiu" em P\&D. Ou seja, nota-se que os percentuais identificados, se aplicados ao faturamento dessas empresas - que em sua maioria foram definidas como sendo de grande (54\%) ou médio (23\%) porte - acaba gerando um volume significativo de recursos financeiros aplicados em $P \& D$. Parece-nos, no entanto, que este montante de recursos financeiros acaba sendo aplicado mais em P\&D interno, sobrando "Poucos recursos financeiros para financiar projetos com a universidade". Enquanto isso, as universidades têm mostrado cada vez mais interesse em captar recursos financeiros para o desenvolvimento de novas pesquisas, pelo fato de este representar o fator mais importante (LEMOS, 2013) para estimular os pesquisadores nas universidades a aumentar suas colaborações com as empresas.

\section{CONSIDERAÇÕES FINAIS}

Os fatores facilitadores e restritivos na cooperação $U-E$, na visão dos líderes de grupos de pesquisa da Udesc que cooperam com as empresas revelam, de um lado, os benefícios proporcionados para a universidade (por exemplo: busca de recursos financeiros adicionais; aquisição ou acesso a recursos materiais e reforço da reputação e da imagem da universidade) e, de outro, as dificuldades (tais como: morosidade da 
universidade pelo excesso de burocracia; docentes sobrecarregados e pouco reconhecimento e valorização dos pesquisadores que atingem resultados positivos nas pesquisas aplicadas).

No que diz respeito às empresas, conclui-se que entre os motivadores centrais na cooperação U-E está o acesso a recursos humanos com elevada qualificação, a localização da universidade e a redução de custos e riscos. Ao repartir custos e riscos entre as duas instituições, a pesquisa cooperativa permite maior investimento na geração de novas tecnologias voltadas a produtos e processos que garantam maior competitividade às organizações e a ampliação do conhecimento científico.

No que se refere aos fatores restritivos, os gestores das empresas pesquisadas destacaram, por exemplo, o excesso de burocracia na universidade; o pouco conhecimento que eles têm em relação às linhas de pesquisa da universidade e o imediatismo por parte da indústria/empresa na busca por resultados. $O$ excesso de burocracia, principalmente nas universidades públicas, devido à complexidade legal, formal e estrutural, revela-se uma das principais barreiras no processo de cooperação U-E e pode gerar conflitos e conduzir o processo à baixa produtividade e eficiência. A estrutura complexa e os processos internos das universidades geram dificuldades como a morosidade na efetivação de contratos e convênios. Outro ponto que merece ser destacado é o desconhecimento do potencial e das capacidades instaladas na universidade por parte das empresas. A visão imediatista e de curto prazo por parte das empresas também revela a necessidade da mudança de postura por parte dos empresários, no sentido de acreditarem mais nas capacidades das universidades, atuando em pesquisas de prazos mais longos e com menor imediatismo de resultados.

Os achados desta pesquisa revelaram os fatores facilitadores e os restritivos à cooperação U-E. Os fatores limitantes devem ser transformados em potencialidades, porque cooperação entre universidade e empresa proporciona a formação de um ambiente colaborativo, estimulando o desenvolvimento tecnológico das empresas e o aprendizado intra e interorganizacional, tanto em conteúdo quanto em procedimentos. Este ambiente colaborativo motiva docentes e alunos a terem um contato maior com os fatos, acontecimentos e rotinas do dia a dia das empresas e do seu pessoal técnico. Ao mesmo tempo, incentiva as empresas a desenvolverem novas tecnologias, fazendo com que estas estejam à frente de seus concorrentes e mais próximas de pesquisadores altamente qualificados, na sua maioria mestres e doutores na área de atuação.

A cooperação U-E não gera benefícios apenas para as universidades e empresas, mas, acima de tudo, para o desenvolvimento regional, seja por meio de seus impactos diretos, vinculados à dinâmica econômica da região, seja por meio de seus impactos indiretos, referentes à difusão e construção do conhecimento e às mudanças socioculturais. As universidades possibilitam a conexão com o mundo, ao mesmo tempo que repercutem de forma multiplicadora nas estruturas espaciais, sobretudo das cidades nas quais estão localizadas, uma vez que o desenvolvimento local está diretamente relacionado com o nível de suporte educacional, e que as desigualdades regionais estão de acordo com os índices educacionais.

É evidente que a universidade contemporânea fortalece sua posição como ator importante no processo de desenvolvimento regional, contribuindo de forma direta e indireta para o desenvolvimento da região em que se insere. As universidades, hoje, 
são motores do crescimento e desenvolvimento socioeconômico, pois as inovações dependem do conhecimento gerado, difundido e aplicado, no entanto deve-se perceber que o papel da universidade neste processo é parcial e depende de outros atores e de um ambiente propício à interação positiva entre estes.

Este artigo tem como limitação o conhecimento que os líderes de Grupos de Pesquisa dos Centros de Ensino (CAV, CCT e Esag), e gestores de empresas pesquisados, possuem acerca do assunto. A pesquisa limitou-se a analisar as parcerias com "Empresas" e, por esta razão, as demais "Instituições parceiras relatadas pelo grupo", no Diretório dos Grupos de Pesquisa do CNPq, não foram consideradas, assim como outros Grupos de Pesquisa de outros Centros de Ensino da Udesc que possuem interação. Os resultados expressam as percepções que os pesquisados têm em relação aos fatores facilitadores e restritivos à cooperação Universidade-Empresa, sendo congruentes quando comparados com os achados de pesquisas já realizadas sobre o assunto.

Os achados do estudo estimulam o desenvolvimento de novas pesquisas, assim como a revisão das políticas e procedimentos utilizados pelas universidades no estabelecimento de parcerias com as empresas. Os fatores restritivos à cooperação U-E podem ser minimizados por meio do diálogo, fóruns de discussões, envolvendo os agentes envolvidos no processo. Uma empresa não pode esperar seis meses para fechar um convênio com a universidade. As universidades em conjunto com as empresas deverão identificar alternativas para desburocratizar os processos internos e, nesse sentido, as fundações de apoio das universidades poderiam sanar os problemas, assim como identificar novas oportunidades.

Novas pesquisas também poderão ser realizadas, visando à identificação das causas e origens das restrições que limitam a formação de parcerias, assim como para entender porque, em muitos casos, os pesquisadores/líderes de grupos de pesquisas estão tão distantes das empresas. Tal situação levanta uma questão que tem e vem incomodando muitos pesquisadores que acreditam na pesquisa aplicada, voltada para a resolução de problemas, como também para alavancar novas oportunidades de aprendizado recíproco.

\section{REFERÊNCIAS}

ALVIM, P. C. R. de C. Cooperação universidade-empresa: da intenção à realidade. In: Interação universidade empresa. Brasília. Instituto Brasileiro de informação em Ciência e Tecnologia, 1998. p. 99124.

ANKRAH, S.; AL-TABBAA, O. Universities-industry collaboration: a systematic review. Scandinavian Journal of Management, v. 31, n. 3, p. 387-408, sept. 2015.

BENEDETTI, M. H.; TORKOMIAN, A. L. V. Uma análise da influência da cooperação Universidade-Empresa sobre a inovação tecnológica. Gestão \& Produção, São Carlos, v. 17, n. 4, p. 145-158, 2010.

BOTELHO, M.; CARRIJO, M.; KAMASAKI, G. Inovações, pequenas empresas e interações com instituições de ensino/pesquisa em arranjos produtivos locais de setores de tecnologia avançada. Revista Brasileira de Inovação, Rio de Janeiro, v. 6, n. 2, p. 331-371, jul./dez. 2007.

CAMARGO, L. C. Cooperação e cooperativismo. São Paulo: Ed. da Universidade de São Paulo, 1960.

CARVALHO, H. G. de. Cooperação com empresas: benefícios para o ensino. 1997. Dissertação (Mestrado) - Centro Federal de Educação Tecnológica do Paraná, Curitiba, 1997.

Cooperação com empresas: benefícios para o ensino. In: INSTITUTO BRASILEIRO DE INFORMA-

ÇÃO EM CIÊNCIA E TECNOLOGIA (IBICT). Interação universidade empresa. Brasília: IBICT, 1998. p. 323358. 
CLARKE, S. Crise do fordismo ou crise da social democracia? Revista Lua Nova, n. 24, p. 117-150, set. 1991.

CLOSS, L. Q.; FERREIRA, G. C. A transferência de tecnologia universidade-empresa no contexto brasileiro: uma revisão de estudos científicos publicados entre os anos 2005 e 2009. Gestão \& Produção, São Carlos, v. 19, n. 2, p. 419-432, 2012.

CONSELHO NACIONAL DE DESENVOLVIMENTO CIENTÍFICO E TECNOLÓGICO (CNPq). 2015. Diretório dos Grupos de Pesquisa no Brasil Lattes. Disponível em: <http://lattes.cnpq.br/web/dgp>. Acesso em: 19 jul. 2015.

DAN, M-C. Why should university and business cooperate? A discussion of advantages and disadvantage. International Journal of Economic Practices and Theories, [S. I], v. 3, n. 1, p. 67-74, jan. 2013.

D'ESTE, P.; PATEL, P. University-industry linkages in the UK: What are the factors underlying the variety of interactions with industry. Research Policy, [S. I], v. 36, n. 9, p. 1.295-1.313, jul. 2007.

DIAS, A. A.; PORTO, G. S. Gestão de transferência da Inova Unicamp. Revista de Administração Contemporânea, Curitiba, v. 17, n. 3, p. 263-284, maio/jun. 2013.

DURHAM, E. R. Avaliação e relações com o setor produtivo. Educação Brasileira, Brasília, v. 7, n. 24, p. 3764, 1990.

ETZKOWITZ, H. The norms of entrepreneurial science: cognitive effects of the new university-industry linkages. Research Policy, [S. I], v. 27, n. 8, p. 823-833, dec. 1998.

The evolution of the entrepreneurial university. International Journal of Technology and Globalisation, [S. I], v. 1, n. 1, p. 64-77, jan. 2004.

FENG, F. et al. Visualization and quantitative study in bibliographic databases: A case in the field of university-industry cooperation\|. Journal of Informetrics, [S. I], v. 9, n. 1, p. 118-134, jan. 2015.

FERREIRA, M. L. A.; RAMOS, R. R. Making university-industry technological partnerships work: a case study in the Brazilian Oil Innovation System. Journal of Technology Management and Innovation, [S. I], v. 10, n. 1, p. 173-187, mar. 2015.

FRANCO, M.; HAASE, H. University-industry cooperation: Researchers' motivations and interaction channels. Journal of Engineering and Technology Management, [S. I], v. 36, p. 41-51, apr./june. 2015.

FREITAS, A. F. de; FREITAS, A. F. de. Interações entre organizações coletivas na promoção do desenvolvimento local. Interações, Campo Grande, v. 14, n. 2, p. 177-188, jul./dez. 2013.

GOEBEL, M. A.; MIURA, M. N. A universidade como fator de desenvolvimento: o caso do município de Toledo-PR. Universidade Estadual do Oeste do Paraná. 2004. Disponível em: <http://e-revista.unioeste.br>. Acesso em: 4 out. 2015.

GUARNICA, L. A.; TORKOMIAN, A. L. V. Gestão de tecnologia em universidades: uma análise do patenteamento e dos fatores de dificuldade e de apoio à transferência de tecnologia no Estado de São Paulo. Gestão \& Produção, São Carlos, v. 16, n. 4, p. 624-638, out./dez. 2009.

KAYMAZ, K.; ERYIĞI, K. Y. Determining factors hindering university-industry collaboration: an analysis from the perspective of academicians in the context of entrepreneurial science paradigm. International Journal of Social Inquiry, [S. I], v. 4, n. 1, p. 85-213, 2011.

KOROTKA, M. A. Proximity factors influencing academics' decisions to cooperate with industrial organizations, Regional Studies. Regional Science, [S. I], v. 2, n. 1, p. 415-423, jul. 2015.

LE, N. X. H. Practices in cooperation between universities and businesses case: lahti university of applied sciences. Bachelor's Thesis, Lhti University of Applied Sciences, Finland, 2015.

LEMOS, D. da C. A interação universidade-empresa para o desenvolvimento inovativo sob a perspectiva institucionalista-evolucionária: uma análise a partir do sistema de ensino superior em Santa Catarina. 2013. Tese (Doutorado) - Universidade Federal de Santa Catarina, Programa de Pós-Graduação em Administração, Florianópolis, 2013.

LOPES, R. P. M. Avaliação do impacto financeiro da universidade pública na economia municipal: o caso de Vitória da Conquista. Revista Conjuntura e Planejamento, Salvador, n. 71, p. 15-21, abr. 2000.

MAIA, M. das G. S. F. A integração universidade/empresa como fator de desenvolvimento regional: um estudo da região metropolitana de Salvador. 2005. Tese (Doutorado) - Departamento de Geografia Física e Análise Geográfica Regional, Faculdade de Geografia e História, Universidade de Barcelona, Barcelona, 2005.

MATHIS, A. Instrumentos para o desenvolvimento sustentável regional. Adcontar, Belém, v. 2, n. 2, p. 1930, 2001.

METCALFE, J. S. University and business relations: connecting the knowledge economy. Minerva, [S. I], v. 48, n. 1, p. 5-33, mar. 2010. 
MIDLEJ, M. M. B. C.; FIALHO, N. H. Universidade e região. Práxis Educacional, Vitória da Conquista, n. 1, p. 171-189, nov. 2005.

NOVELI, M.; SEGATTO, A. P. Processo de cooperação universidade empresa para a inovação tecnológica em um parque tecnológico: evidências empíricas e proposição de um modelo conceitual. Revista de Administração e Inovação, São Paulo, v. 9, n. 1, p. 81-105, jan./mar. 2012.

PERKMANN, M.; NEELY, A.; WALSH, K. How should firms evaluate success in university-industry alliances? A performance measurement system. R\&D Management, [S. I], v. 41, n. 2, p. 202-216, feb. 2011.

PERKMANN, M.; SCHILDT, H. Open data partnerships between firms and universities: The role of boundary organizations. Research Policy, [S. I], v. 38, n. 5, p. 1-11, june. 2014.

PHILBIN, S. P. An investigation of the development and management of university research institutes. Journal of Research Administration, [S. I], v. 42, n. 1, p. 103-122, 2011.

PLEWA, C. et al. University-Industry linkage evolution: an empirical investigation of relational success factors. R\&D Management, [S. I], v. 43, n. 4, p. 365-380, 2013.

PLONSKI, G. A. Cooperação empresa-universidade no Brasil: um novo balanço prospectivo. In:

(Coord.). Interação universidade-empresa. Brasília: IBICT, 1998. p. 9-23. V. 1.

PORTO, G. Características do processo decisório na cooperação empresa-universidade. Revista de Administração Contemporânea, Curitiba, v. 8, n. 3, p. 29-52, jul./set., 2004.

A decisão de cooperação universidade - empresa sob a ótica dos líderes de grupos de pesquisa da USP cadastrados no diretório de pesquisa do CNPq. 2006. Tese (Livre docência) - Universidade de São Paulo, Ribeirão Preto, SP, 2006.

QUANDT, C. O. Redes de cooperação e inovação localizada: estudo de caso de um Arranjo Produtivo Local. Revista de Administração e Inovação, São Paulo, v. 9, n. 1, p. 141-166, jan./mar. 2012.

Inovação tecnológica. In: SILVA JÚNIOR, R. (Org.). Empreendedorismo tecnológico. Instituto de Engenharia do Paraná: Curitiba, 2009. p. 71-101.

RANGA, M. et al. University-Industry Cooperation in the US and Canada. European Commission, DG Education and Culture, 2013.

RIBEIRO, R. J.; ANDRADE, E. P. Modelo de gestão para incubadora de empresas sob a perspectiva de metodologias. Organização \& Estratégia, v. 4, n. 1, p. 71-90, jan./abr. 2008.

ROLIM. C.; SERRA, M. Universidade e desenvolvimento regional - o apoio das Instituições de Ensino Superior ao desenvolvimento regional. Curitiba: Juruá, 2009.

ROSA, R. A. et al. Cooperação Universidade-Empresa: um estudo bibliométrico e sociométrico em periódicos científicos brasileiros de administração. LATIN IBERO-AMERICAN CONFERENCE ON MANAGEMENT OF TECHNOLOGY, 16., 2015. Porto Alegre, out. 2015. p. 1-20. V. 1.

SANTANA, E. E. de P.; PORTO, G. S. E agora, o que fazer com essa tecnologia? Um estudo multicaso sobre as possibilidades de transferência de tecnologia na USP-RP. Revista de Administração Contemporânea, Curitiba, v. 13, n. 3, p. 410-429, jul./ago. 2009.

SCHNEIDER, L. Educação e desenvolvimento: um estudo do impacto econômico da universidade federal no município de Santa Maria (RS). Santa Maria: Unifra, 2002.

SEGATTO, A. P. Análise do processo de cooperação tecnológica universidade - empresa: um estudo exploratório. 1996. Dissertação (Mestrado) - Universidade de São Paulo, Faculdade de Economia, Administração e Contabilidade, São Paulo, 1996.

SEGATTO-MENDES, A, P.; MENDES, N. Cooperação tecnológica universidade-empresa para eficiência energética: um estudo de caso. Revista de Administração Contemporânea, Curitiba, v. 10, n. especial, p. 53-75, 2006.

SILVA, C. V. Processo de transferência de conhecimento na interação universidade-empresa: programas de incubação do Distrito Federal. Revista Ibero-Americana de Ciência da Informação, Brasília, v. 8, n. 1, p. 94-95, 2015.

Processo de transferência de conhecimento na interação universidade - empresa: programas de incubação do Distrito Federal. Brasília: UnB, 2010.

SILVEIRA, L. A. Relação universidade-empresa: fatores propulsores e restritivos no processo de transferência de tecnologia nas empresas catarinenses. 2005. Tese (Doutorado) - Universidade Federal de Santa Catarina, Engenharia de Produção, Florianópolis, 2005.

STAL, E.; FUJINO, A. As relações universidade-empresa no Brasil sob a ótica da Lei de Inovação. Revista de Administração e Inovação, São Paulo, v. 2, n. 1, p. 5-19, 2005.

TECCHIO, E. L. et al. Cooperação universidade-segmento empresarial: a realidade da Universidade Federal de Santa Catarina. Desenvolvimento em Questão, ljuí: Ed. Unijuí, v. 11, n. 22, p. 173-207, jan./abr. 2013. 
TEIXEIRA, A. Bases preliminares para o plano de educação referente ao Fundo Nacional de Ensino Primário. Revista Brasileira de Estudos Pedagógicos, Rio de Janeiro, v. 38, n. 88, p. 97-107, out./dez. 1962.

TORKOMIAN, A. L. V. Gestão de tecnologia na pesquisa acadêmica: o caso de São Carlos. 1997. Tese (Doutorado) - Universidade de São Paulo, Faculdade de Economia, Administração e Contabilidade, São Paulo, 1997.

UNIVERSIDADE DO ESTADO DE SANTA CATARINA (Udesc). Avaliação do MEC reafirma que Udesc é quarta melhor estadual do Brasil e 18므 no geral. Florianópolis, 2014. Disponível em: <http://www.udesc.br/?idNoticia=12180>. Acesso em: 20 dez. 2014.

Resolução 029/2009 do Conselho Universitário da Udesc. Estabelece normas para a ocupação docente na Udesc. Florianópolis, 2009. Disponível em: <http://www.secon.udesc.br>. Acesso em: 20 jun. 2015.

Udesc em números. Disponível em: <http://www.udesc.br/numeros>. Acesso em: 14 out. 2017. VIEIRA, E. F.; VIEIRA, M. M. F. Funcionalidade burocrática nas universidades federais: conflito em tempos de mudança. Revista de Administração Contemporânea, Curitiba, v. 8, n. 2, p. 181-200, abr./jun. 2004.

WEBSTER, A. J.; ETZKOWITZ H. Academic-Industry relations: the second academic revolution. London: Science Policy Support Group, 1991. (SPSG concept paper n. 12). 